\title{
NÍVEIS DE LISINA PARA FRANGOS DE CORTE NOS PERÍODOS DE 22 A 42 E DE 43 A 49 DIAS DE IDADE ${ }^{1}$
}

\author{
Lysine levels for broilers in the periods from 22 to 42 and 43 to 49 days of age
}

\author{
Fernando Guilherme Perazzo Costa ${ }^{2}$, Valene da Silva Amarante Junior ${ }^{3}$, Germano Augusto Jerônimo do Nascimento ${ }^{4}$, \\ Patrícia Araújo Brandão ${ }^{3}$, Leilane Rocha Barros ${ }^{4}$, José Humberto Vilar da Silva ${ }^{5}$, Janaíne Sena da Costa ${ }^{6}$
}

\begin{abstract}
RESUMO
Foram realizados dois experimentos para determinar a exigência de lisina total para frangos de corte Ross, macho, no período de 22 a 42 e de 43 a 49 dias de idade. Utilizou-se em cada experimento, 900 frangos com peso médio inicial de $764 \mathrm{~g} \pm 35 \mathrm{~g}$ aos 22 dias e de $2520 \pm 73 \mathrm{~g}$ aos 43 dias de idade. As aves foram distribuídas em um delineamento inteiramente casualizado, com seis tratamentos, seis repetições e 25 aves por unidade experimental. Os tratamentos foram constituídos de seis níveis lisina, de acordo com a fase. As exigências nutricionais mínimas, exceto para lisina, foram atendidas. As variáveis avaliadas foram consumo de ração, ganho de peso, conversão alimentar, rendimento de carcaça e dos principais cortes, porcentagem de gordura abdominal, coração, fígado e moela, sendo as exigências de lisina estimadas por modelos de regressão. Considerando os dados obtidos, as exigências nutricionais de lisina total foram estimadas em 1,111\% para o máximo desempenho, no período 22 a 42 dias de idade e de 1,044\% para o máximo desempenho e rendimento de carcaça e peito, no período de 43 a 49 dias de idade.
\end{abstract}

Termos para indexação: Aminoácidos, carcaça, desempenho, exigências, frangos de corte.

\begin{abstract}
Two experiments were carried out to determine the requirement of total lysine for male Ross broilers from 22 to 42 and 43 to 49 days of age. A total of 900 broilers with initial body weight of $764 \pm 35 \mathrm{~g}$ at 22 days and $2520 \pm 73 \mathrm{~g}$ at 43 days of age. The broilers were distributed in a completely randomized experimental design with six treatments, six replicates and 25 birds per experimental unit. There were six synthetic lysine levels in each phase. Minimum nutritional requirements were attended, except for lysine. Feed intake, body weight gain, feed conversion, carcass yield and the main cuts were evaluated lysine requirements were estimated by using regression models. Considering the performance data, the nutritional requirements of total lysine were $1.111 \%$ for maximum performance from 22 to 42 days of age and $1.044 \%$ for maximum performance and carcass and breast yields from 43 to 49 days of age.
\end{abstract}

Index terms: Amino acids, broilers, carcass, performance, requirement.

(Recebido para publicação em 3 de março de 2005 e aprovado em 31 de janeiro de 2006)

\section{INTRODUÇÃO}

A exigência dos aminoácidos é estimada com base em um aminoácido referência. $\mathrm{O}$ aminoácido usado tem sido a lisina, cuja escolha é devida principalmente ao fato de que, em seu metabolismo, é usada quase que exclusivamente para acréscimo de proteína corporal (PACK, 1995). O conceito de proteína ideal tem sido utilizado como um modelo para maximizar o aproveitamento protéico, através do balanço adequado de todos os aminoácidos essenciais. No entanto, torna-se necessário melhor conhecimento dos requerimentos nutricionais das aves, pois um excesso de aminoácidos corresponde em degradação da proteína, sendo o nitrogênio $(\mathrm{N})$ eliminado pela urina (SCOTT et al., 1982; SUIDA, 2001).
A lisina por ser considerado o segundo aminoácido limitante para frangos de corte, tem tido sua exigência largamente estudada. No entanto, diversos fatores podem afetar a exigência de lisina, como estresse, linhagem, ambiente térmico, teor de proteína da ração, energia metabolizável e, principalmente, os alimentos utilizados nas formulações (CONHALATO, 1998).

Entretanto, a exigência de aminoácidos e proteína para aves varia de acordo com o estágio de produção (SILVA et al., 1999). E, ainda dentro de uma mesma fase de produção pode haver variações, observando-se que os níveis encontrados para ganho de peso diferenciam dos encontrados para conversão alimentar, relacionada ao custo alimentar e rendimento de peito (NASCIMENTO, 2003a).

\footnotetext{
${ }^{1}$ Parte da Tese de Doutorado do segundo autor.

${ }^{2}$ Professor do Departamento de Zootecnia do Centro de Ciências Agrárias/CCA - Universidade Federal da Paraíba/UFPB - Cx. P. 9 - 58.397-000 Areia, PB - fperazzo@cca.ufpb.br

${ }^{3}$ Aluno do Programa de Doutorado Integrado em Zootecnia (UFRPE/UFC/UFPB).

${ }^{4}$ Aluno do Programa de Pós-Graduação em Zootecnia CCA - Universidade Federal da Paraíba/UFPB - Cx. P. 9 - 58.397-000 - Areia, PB.

${ }^{5}$ Professor do Centro de Formação de Tecnólogos CFT - Universidade Federal da Paraíba/UFPB.

${ }^{6}$ Aluno do Curso de Zootecnia do CCA - Universidade Federal da Paraíba/UFPB - Cx. P. 9 - 58.397-000 - Areia, PB.
} 
A utilização de aminoácidos sintéticos nas rações tem proporcionado facilidade no ajuste das formulações, por possibilitar melhor balanço entre os aminoácidos essenciais. Além disso, as formas sintéticas dos principais aminoácidos limitantes estão comercialmente disponíveis a preços que cada ano os tornam mais competitivos em relação aos custos dos aminoácidos presentes nos alimentos (ROSTAGNO et al., 2004).

As recomendações dos níveis dietéticos de lisina, para frangos de corte na fase de crescimento ( 29 a 42 dias) determinada por Rezende et al. (1980) é de $0,75 \%$ nas rações com $16 \%$ de proteína bruta. O NRC (1994) recomenda 1,00 e $0,85 \%$ de lisina total para a fase de 22 a 42 e 43 a 56 dias, em rações com $3.200 \mathrm{kcal}$ de EM/kg, respectivamente. Já Rostagno et al. (2000) recomendam 1,156 e 1,040 \% de lisina total para a fase de 22 a 42 e 43 a 49 dias, em rações com 3.100 e $3.200 \mathrm{kcal} \mathrm{de} \mathrm{EM} / \mathrm{kg}$. Considerando-se o desenvolvimento tecnológico, principalmente da genética destas aves nas ultimas décadas, a pesquisa na área da nutrição teve que acompanhar esta evolução, verificando-se um aumento nos valores das exigências de lisina para frangos de corte.

Em geral, verificam-se consideráveis variações quanto aos valores de exigência de lisina (AL-NASSER et al., 1986). Por isso, a determinação das exigências nutricionais desses aminoácidos para frangos de corte ainda é fator de grande importância para a moderna avicultura industrial.

Portanto, o presente trabalho foi conduzido para avaliar o desempenho de frangos de corte nos períodos de 22 a 42 e de 43 a 49 dias de idade, submetidos a dietas contendo diferentes níveis de lisina.

\section{MATERIAL E MÉTODOS}

Os experimentos foram conduzidos no Aviário Experimental do Departamento de Zootecnia do Centro de Ciências Agrárias da Universidade Federal da Paraíba. Os frangos foram alojados em um galpão de alvenaria, com 36 boxes na densidade de $10 \mathrm{aves} / \mathrm{m}^{2}$. Durante o período experimental, adotou-se um programa de luz contínua (24 horas de luz natural + artificial). As leituras de temperatura e umidade máximas e mínimas foram registradas diariamente através de um termômetro de bulbo seco e úmido.

Durante os períodos pré-inicial (1 a 7 dias) e inicial (8 a 21 dias), as aves receberam rações comerciais à base de milho e farelo de soja, com 23 e $21 \%$ de PB e 2930 e 3050 $\mathrm{kcal} / \mathrm{kg}$ de EM, respectivamente. A água e a ração foram fornecidas à vontade.

Em cada fase avaliada ( 22 a 42 e 43 a 49 dias), foram utilizados 900 frangos de corte, Ross, machos, em um delineamento experimental inteiramente casualizado, com seis tratamentos (seis níveis de lisina), seis repetições e 25 aves por unidade experimental. Os pesos iniciais das aves foram de $764 \mathrm{~g}$ para a fase de 22 a 42 e $2.520 \mathrm{~g}$ para a fase de 43 a 49 dias. As aves receberam duas dietas deficientes em lisina (Tabela 1), uma contendo 19,5\% de PB e $3.100 \mathrm{Kcal}$ de EM/Kg suplementadas com seis níveis de lisina $(0,00 ; 0,080 ; 0,160$; 0,$240 ; 0,320$ e $0,400 \%$ ), correspondendo aos níveis de 0,936 ; 1,$016 ; 1,096 ; 1,176 ; 1,256$ e 1,336\% de lisina total na ração no período de 22 a 42 dias e a outra com e $18 \%$ de PB e $3.200 \mathrm{Kcal}$ de EM/Kg, correspondendo aos níveis de 0,850; 0,930; 1,010; 1,$090 ; 1,170$ e 1,250\% de lisina total nas rações, no período de 43 a 49 dias. As exigências nutricionais mínimas, exceto para lisina, foram atendidas segundo as recomendações de Rostagno et al. (2000), e as suplementações com L-lisina HCl (98\%), contendo 79,2\% de lisina, considerando-a com $100 \%$ de digestibilidade verdadeira (LECLERCQ, 1998), foram realizadas em substituição ao amido de milho na ração.

Ao final de cada experimento em que se utilizou animais diferentes, avaliou-se o consumo de ração, o ganho de peso e a conversão alimentar. Após um período de jejum de oito horas, foram abatidas quatro aves por unidade experimental, com peso médio da repetição, para a determinação dos rendimentos de carcaça em relação ao peso vivo das aves ao abate, enquanto que os rendimentos de peito, coxa, sobre-coxa, peso do coração, fígado, moela e gordura abdominal em relação à carcaça eviscerada (cabeça, pescoço e vísceras).

As análises estatísticas foram realizadas através de análise de variância para as características avaliadas e por modelo de regressão polinomial para as estimativas da exigência de lisina. $O$ programa computacional utilizado foi o SAS (SAS INSTITUTE, 1996).

\section{RESULTADOS E DISCUSSÃO}

Efeito dos níveis de lisina sobre o desempenho e avaliação de carcaça de frangos de corte no período de 22 a 42 dias de idade.

$\mathrm{Na}$ Tabela 2, estão apresentadas as médias dos dados de desempenho dos frangos.

Houve efeito linear dos níveis de lisina sobre o consumo de ração dos frangos $\left(v=1,5661+0,2859 X, R^{2}=\right.$ $0,75)$. Este resultado é contrário àqueles obtidos por Borges et al. (2002) e Valério et al. (2003), que não observaram variação no consumo de ração das aves, em função dos níveis de lisina na ração. Para ganho de peso houve efeito quadrático $(\mathrm{P}<0,05)$ dos níveis de lisina, cujo ganho de peso aumentou até o nível de $1,102 \%$, conforme pode ser observado na Figura 1. 
TABELA 1 - Composição percentual da dieta basal ${ }^{1}$.

\begin{tabular}{|c|c|c|}
\hline \multirow[t]{2}{*}{ Ingredientes } & \multicolumn{2}{|c|}{ Ração experimental } \\
\hline & 22-42 dias & 43-49 dias \\
\hline Milho & 65,180 & 62,588 \\
\hline Farelo de soja & 25,590 & 23,986 \\
\hline Glúten de milho & 2,583 & 4,531 \\
\hline Amido & 2,300 & 2,672 \\
\hline Fosfato bicálcico & 1,586 & 2,500 \\
\hline Calcário & 0,978 & 1,433 \\
\hline Óleo de soja & 0,818 & 0,906 \\
\hline Premix vitamínico ${ }^{2}$ & 0,100 & 0,060 \\
\hline Premix mineral $^{3}$ & 0,050 & 0,050 \\
\hline Bacitracina de zinco & 0,020 & 0,020 \\
\hline Coban $200^{4}$ & 0,060 & 0,050 \\
\hline Sal comum & 0,425 & 0,800 \\
\hline $\mathrm{BHT}^{5}$ & 0,010 & 0,010 \\
\hline Inerte $^{6}$ & 0,300 & 0,394 \\
\hline Total & 100,00 & 100,00 \\
\hline \multicolumn{3}{|l|}{ Valores nutricionais calculados } \\
\hline Energia metabolizável (kcal/kg) & 3.100 & 3.200 \\
\hline Proteína bruta (\%) & 19,50 & 18,000 \\
\hline Cálcio (\%) & 0,874 & 0,800 \\
\hline Fósforo disponível (\%) & 0,406 & 0,365 \\
\hline Lisina total $(\%)$ & 0,936 & 0,850 \\
\hline Metionina+cistina total (\%) & 0,664 & 0,603 \\
\hline Treonina total (\%) & 0,751 & 0,690 \\
\hline Arginina total (\%) & 1,186 & 1,101 \\
\hline Triptofano total (\%) & 0,215 & 0,201 \\
\hline Valina total (\%) & 0,898 & 0,838 \\
\hline Sódio (\%) & 0,192 & 0,192 \\
\hline
\end{tabular}

${ }^{1}$ Valores calculados de acordo com Rostagno et al. (2000). ${ }^{2}$ Premix vitamínico por kg de ração - Vit. A - 15.000.000 Ul, Vit. $\mathrm{D}_{3}$ - 1.500.000 Ul, Vit. E - 15.000 Ul, Vit.B - 2,0 g, Vit.B $-4,0 \mathrm{~g}$, VitB6 - 3,0 g, Vit.B ${ }_{12}$ - 0,015 g, Ácido nicotínico - 25 g, Ácido pantotênico - $10 \mathrm{~g}$, Vit. $\mathrm{K}_{3}-3,0 \mathrm{~g}$, Ácido fólico - $1,0 \mathrm{~g}$, Bacitracina de zinco - $10 \mathrm{~g}$, Selênio - $250 \mathrm{mg},{ }^{3}$ Premix mineral por kg de ração - Mn,60 g; Fe, 80 g; Zn,50 g; Cu, 10 g; Co, 2 g; I, 1 g; e veículo q.s.p.,500 g.; ${ }^{4}$ Monensina sódica, 200 g/ton.; ${ }^{5}$ Antioxidante BHT - $10 \mathrm{~g}$, e veículo q.s.p. - $1.000 \mathrm{~g} ;{ }^{6}$ Inerte- areia lavada. 
TABELA 2 - Desempenho dos frangos de corte alimentados com diferentes níveis de lisina, no período entre 22 e 42 dias de idade.

\begin{tabular}{cccc}
\hline $\begin{array}{c}\text { Níveis de Lisina } \\
(\boldsymbol{\%})\end{array}$ & Consumo de Ração $(\mathbf{k g})$ & $\begin{array}{c}\text { Ganho de Peso } \\
(\mathbf{k g})\end{array}$ & $\begin{array}{c}\text { Conversão alimentar } \\
(\mathbf{k g} / \mathbf{k g})\end{array}$ \\
\hline 0,936 & 2,928 & 1,567 & 1,868 \\
1,016 & 2,902 & 1,588 & 1,829 \\
1,096 & 2,944 & 1,607 & 1,832 \\
1,176 & 3,032 & 1,585 & 1,913 \\
1,256 & 3,024 & 1,567 & 1,931 \\
1,336 & 3,006 & 1,547 & 1,955 \\
\hline Média & 2,973 & 1,577 & 1,888 \\
Regressão & $\mathrm{L}^{*}$ & $\mathrm{Q}^{*}$ & $\mathrm{~L}^{*}$ \\
\hline CV $(\%)$ & 2,48 & 2,18 & 3,11 \\
\hline
\end{tabular}

$\mathrm{CR}=$ Consumo de Ração

$\mathrm{GP}=$ Ganho de peso

$\mathrm{CA}=$ Conversão alimentar

$\mathrm{Q}^{*}=$ Efeito Quadrático $(\mathrm{P}<0,05)$

$\mathrm{L}^{*}=$ Efeito Linear $(\mathrm{P}<0,05)$

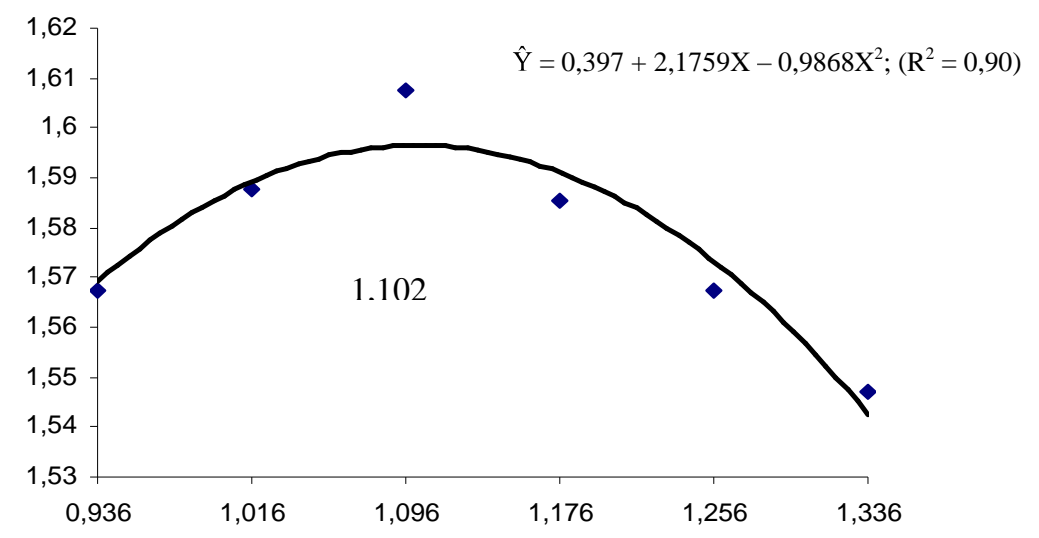

FIGURA 1 - Ganho de Peso de frangos de corte alimentados com diferentes níveis lisina, no período de 22 a 42 dias de idade.

Este resultado é inferior a 1,12 e 1,20\% de lisina total obtido por Conhalato (1998) e Valério et al. (2003), respectivamente, enquanto é superior a $0,93 \%$ de lisina total obtido por Han \& Baker (1994). Por outro lado, Costa (2000) não observou efeito dos níveis de lisina sobre o ganho de peso dos frangos. Com relação à conversão alimentar, constatou-se um aumento linear $(\mathrm{P}<0,01)$ quando os níveis de lisina na ração foram aumentados $(\mathrm{v}=1,5661$ $+0,2859 X, R^{2}=0,75$ ).

Entretanto, Conhalato (1998), Costa (2000) e Valério et al. (2003), verificaram uma melhor conversão alimentar de frangos de corte em função dos diferentes níveis de lisina das rações, que segundo Nascimento (2003b) ocorre pela maior ingestão de lisina da dieta, promovendo um 
maior crescimento muscular e ganho de peso, diminuindo a gordura e não alterando o consumo de ração, favorecendo desta maneira uma maior otimização da conversão, o que não foi verificado no presente experimento, provavelmente provocado pelo elevado consumo de ração.

Na Tabela 3, estão apresentadas as médias dos dados de avaliação de carcaça. Não houve efeito(P>0,05) dos níveis de lisina sobre os rendimentos de carcaça, peito, coxa, sobrecoxa, coração e fígado. Os resultados de rendimento de carcaça se assemelham aos obtidos por Valério et al. (2003), que também não encontraram efeito dos níveis de lisina sobre o peso relativo de coxa e sobre-coxa.

Os níveis de lisina na ração influenciaram $(\mathrm{P}<0,01)$ o peso relativo da gordura abdominal, que reduziu de forma quadrática até o nível de $1,111 \%$ de lisina, conforme a equação $\mathrm{v}=1,0112-4,2669 \mathrm{X}+1,9206 \mathrm{X}^{2}, \mathrm{R}^{2}=0,91$. Este resultado se aproxima com o obtido por Borges et al. (2002) que observaram uma redução quadrática da gordura abdominal até o nível de 1,00 \% de lisina. Já Valério et al. (2003) observaram uma redução linear e Barboza (1998) não constatou efeito dos níveis de lisina sobre o conteúdo da gordura abdominal dos frangos.

A exigência de $1,111 \%$ de lisina para a mínima deposição de gordura abdominal em relação ao ganho de peso (1,102\% de lisina) está de acordo com Leclercq (1998) que afirma que a exigência para mínima deposição de gordura animal é mais elevada do que para o ganho de peso, conversão alimentar e rendimento de peito. Constatou-se uma redução quadrática $(\mathrm{P}<0,05)$ até o nível $1,131 \%$ de lisina no peso relativo da moela dos frangos (v $\left.=3,7431-3,4394 \mathrm{X}+1,5206 \mathrm{X}^{2}, \mathrm{R}^{2}=0,98\right)$. Farrel \& Koong (1986) associaram a redução na exigência de mantença à diminuição da massa e conseqüentemente da atividade metabólica dos órgãos internos de ratos em crescimento. Esse resultado é contrário ao obtido por Valério et al. (2003) que observaram redução linear no peso da moela de frangos com o acréscimo dos níveis de lisina. Diante do resultado observado nessa pesquisa, evidenciou que o crescimento da moela pode ter sido influenciado pelo nível de lisina através da síntese de proteína muscular.

Efeito dos níveis de lisina da ração sobre o desempenho e avaliação de carcaça de frangos de corte no período de 43 a 49 dias de idade.

Na Tabela 4 estão apresentados os valores médios do desempenho dos frangos. Houve efeito quadrático $(\mathrm{P}<0,01)$ dos níveis de lisina sobre o consumo de ração (CR), o qual aumentou até o nível de $1,097 \%$ de lisina total $\left(\mathrm{v}=0,1858+1,9117 \mathrm{X}-0,871 \mathrm{X}^{2} ; \mathrm{R}^{2}=0,68\right)$. Estes resultados se assemelham aos obtidos por Skinner et al. (1992) que verificaram um aumento no consumo de ração dos frangos de corte no período de 42 a 49 dias de idade. Porém, Cella et al. (2001) não encontraram efeito dos níveis de lisina sobre o consumo das aves.

TABELA 3 - Valores de pesos relativos da avaliação de carcaça de frangos de corte, alimentados com diferentes níveis de lisina de 22 a 42 dias de idade.

\begin{tabular}{ccccccccc}
\hline $\begin{array}{l}\text { Níveis de } \\
\text { lisina (\%) }\end{array}$ & $\begin{array}{c}\text { Carcaça } \\
(\boldsymbol{\%})\end{array}$ & $\begin{array}{c}\text { Peito } \\
(\boldsymbol{\%})\end{array}$ & $\begin{array}{c}\text { Coxa } \\
(\boldsymbol{\%})\end{array}$ & $\begin{array}{c}\text { Sobre- } \\
\mathbf{c o x a}(\boldsymbol{\%})\end{array}$ & $\begin{array}{c}\text { Gordura } \\
\text { Abdominal }(\boldsymbol{\%})\end{array}$ & $\begin{array}{c}\text { Coração } \\
(\boldsymbol{\%})\end{array}$ & $\begin{array}{c}\text { Fígado } \\
(\boldsymbol{\%})\end{array}$ & $\begin{array}{c}\text { Moela } \\
(\boldsymbol{\%})\end{array}$ \\
\hline 0,936 & 75,56 & 28,45 & 12,41 & 15,43 & 1,70 & 0,62 & 2,35 & 1,80 \\
1,016 & 76,19 & 28,76 & 12,55 & 15,67 & 1,66 & 0,63 & 2,31 & 1,82 \\
1,096 & 76,84 & 29,02 & 12,74 & 15,91 & 1,62 & 0,65 & 2,34 & 1,84 \\
1,176 & 76,21 & 28,85 & 14,98 & 15,96 & 1,66 & 0,66 & 2,35 & 1,90 \\
1,256 & 75,57 & 28,56 & 13,04 & 16,17 & 1,70 & 0,64 & 2,36 & 1,82 \\
1,336 & 74,91 & 28,30 & 13,08 & 16,41 & 1,73 & 0,63 & 2,34 & 1,79 \\
\hline Regressão & NS & NS & NS & NS & Q** & NS & NS & Q* \\
CV (\%) & 3,27 & 4,47 & 3,47 & 4,99 & 2,92 & 7,38 & 1,87 & 2,61 \\
\hline
\end{tabular}

$\mathrm{NS}=$ não-significativo pelo teste $\mathrm{F}(\mathrm{P}>0,05)$.

$\mathrm{Q}^{*}$ e $\mathrm{Q}^{* *}=$ Efeito Quadrático $(\mathrm{P}<0,05$ e $\mathrm{P}<0,01)$. 
TABELA 4 - Desempenho de frangos de corte alimentados com diferentes níveis de lisina, no período de 43 a 49 dias de idade.

\begin{tabular}{cccc}
\hline $\begin{array}{c}\text { Níveis de Lisina } \\
(\boldsymbol{\%})\end{array}$ & Consumo de Ração $\mathbf{( k g )}$ & $\begin{array}{c}\text { Ganho de Peso } \\
(\mathbf{k g})\end{array}$ & $\begin{array}{c}\text { Conversão alimentar } \\
(\mathbf{k g} / \mathbf{k g})\end{array}$ \\
\hline 0,850 & 1,166 & 0,524 & 2,263 \\
0,930 & 1,216 & 0,550 & 2,211 \\
1,010 & 1,251 & 0,578 & 2,167 \\
1,090 & 1,232 & 0,557 & 2,216 \\
1,171 & 1,223 & 0,540 & 2,268 \\
1,250 & 1,222 & 0,529 & 2,322 \\
\hline Média & 1,218 & 0,546 & 2,241 \\
Regressão & $\mathrm{Q}^{* *}$ & $\mathrm{Q} *$ & $\mathrm{NS}$ \\
\hline CV (\%) & 2,06 & 7,61 & 7,50 \\
\hline
\end{tabular}

$\mathrm{CR}=$ Consumo de Ração.

$\mathrm{GP}=$ Ganho de peso.

$\mathrm{CA}=$ Conversão alimentar.

Q* e $\mathrm{Q}^{* *}=$ Efeito Quadrático $(\mathrm{P}<0,05$ e $\mathrm{P}<0,01)$.

$\mathrm{NS}=$ não-significativo.

Este efeito quadrático para o consumo de ração justifica-se, pelo fato de que a ave para atingir o ponto de máxima exigência, aumenta o consumo para satisfazer sua necessidade e após o ponto máximo vai decrescendo o consumo pelo fato de que a necessidade energética vai diminuindo. Entretanto, o excesso de aminoácido na ração, além da necessidade de síntese protéica, é degradado e o nitrogênio oriundo dessa degradação é eliminado através da urina, sendo que o esqueleto de carbono que sobra pode ser usado na síntese de glicose, convertido em gordura ou em $\mathrm{CO}_{2}$ e $\mathrm{H}_{2} \mathrm{O}$ (SCOTT et al., 1982), promovendo dessa forma um incremento no metabolismo energético.

Houve efeito quadrático $(\mathrm{P}<0,05)$ dos níveis de lisina sobre o ganho de peso, o qual apresentou uma exigência de 1,044 \% de lisina total (Figura 2). Este resultado é superior aos $0,90 \%$ de lisina total recomendados por Barboza et al. (2000) e semelhante ao nível de 1,040\%, recomendado por Rostagno et al. (2000).

Não houve efeito dos níveis de lisina na ração sobre o rendimento de carcaça, coxa, sobre-coxa, gordura abdominal, fígado, coração e moela, conforme pode ser observado na Tabela 5 .

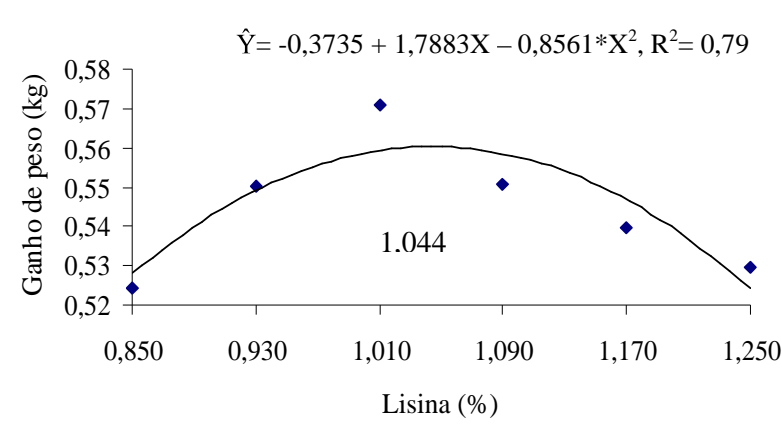

FIGURA 2 - Ganho de peso de frangos de corte submetidos a diferentes níveis de lisina, no período de 43 a 49 dias de idade.

Os níveis de lisina na ração influenciaram $(\mathrm{P}<0,01)$ o rendimento de peito, que aumentou linearmente, conforme a equação $v=142,74+28,407 X ; R^{2}=0,96$. Este resultado corrobora com os obtidos por Costa (2000). Este aumento está relacionado ao fato de que a lisina é considerada um aminoácido de deposição de proteína corporal e, no caso de peito, seria mais bem aproveitada devido aos tipos de fibras musculares que este possui (LECLERCQ, 1998). 
TABELA 5 - Valores de pesos relativos da avaliação de carcaça de frangos de corte alimentados com diferentes níveis de lisina, no período de 43 a 49 dias de idade.

\begin{tabular}{ccccccccc}
\hline $\begin{array}{c}\text { Níveis } \\
\text { lisina }(\boldsymbol{\%})\end{array}$ & $\begin{array}{c}\text { Carcaça } \\
(\boldsymbol{\%})\end{array}$ & $\begin{array}{c}\text { Peito } \\
(\boldsymbol{\%})\end{array}$ & $\begin{array}{c}\text { Coxa } \\
(\boldsymbol{\%})\end{array}$ & $\begin{array}{c}\text { Sobre-coxa } \\
(\boldsymbol{\%})\end{array}$ & $\begin{array}{c}\text { Gordura } \\
\text { Abdominal }(\boldsymbol{\%})\end{array}$ & $\begin{array}{c}\text { Coração } \\
(\boldsymbol{\%})\end{array}$ & $\begin{array}{c}\text { Fígado } \\
(\boldsymbol{\%})\end{array}$ & $\begin{array}{c}\text { Moela } \\
(\boldsymbol{\%})\end{array}$ \\
\hline 0,850 & 81,26 & 27,70 & 12,22 & 14,98 & 2,25 & 0,44 & 1,54 & 1,25 \\
0,930 & 81,15 & 28,40 & 12,27 & 14,69 & 2,33 & 0,44 & 1,56 & 1,23 \\
1,010 & 84,48 & 28,61 & 12,34 & 14,79 & 2,38 & 0,42 & 1,62 & 1,23 \\
1,090 & 81,13 & 28,78 & 12,38 & 14,52 & 2,16 & 0,42 & 1,51 & 1,19 \\
1,170 & 81,47 & 29,30 & 12,41 & 14,23 & 2,33 & 0,41 & 1,61 & 1,19 \\
1,250 & 81,52 & 29,78 & 12,44 & 14,69 & 2,15 & 0,41 & 1,58 & 1,25 \\
\hline Média & 81,84 & 28,76 & 12,34 & 14,65 & 2,27 & 0,42 & 1,57 & 1,22 \\
Regressão & $\mathrm{NS}$ & $\mathrm{L} * *$ & $\mathrm{NS}$ & $\mathrm{NS}$ & $\mathrm{NS}$ & $\mathrm{NS}$ & $\mathrm{NS}$ & $\mathrm{NS}$ \\
\hline CV (\%) & 3,30 & 2,66 & 2,94 & 3,51 & 11,59 & 8,05 & 5,04 & 5,62 \\
\hline
\end{tabular}

$\mathrm{L}^{* *}=$ Efeito linear $(\mathrm{P}<0,01)$.

$\mathrm{NS}=$ não-significativo $(\mathrm{P}>0,05)$.

\section{CONCLUSÃO}

Os dados de desempenho e avaliação de carcaça recomendam o nível nutricional de $1,111 \%$ de lisina total para frangos de corte, machos, no período de 22 a 42 e $1,044 \%$ de lisina total para a fase de 43 a 49 dias de idade.

\section{REFERÊNCIAS BIBLIOGRÁFICAS}

AL-NASSER, A. Y.; ILIAN-AWADI, A. A.; DIAB, M. F. The effect of adding essential amino acids and vitamins to ration of broilers. Poultry Science, Champaign, v. 65, p. 742-748, 1986.

BARBOZA, W. A. Exigências nutricionais de lisina para duas marcas comerciais de frangos de corte. 1998. $116 \mathrm{f}$. Tese (Doutorado em Zootecnia) - Universidade Federal de Viçosa, Viçosa, 1998.

BARBOZA, W. A.; ROSTAGNO, H. S.; ALBINO, L. F. T. Níveis de lisina para frangos de corte de 22 a 40 e 42 a 48 dias de idade. Revista Brasileira de Zootecnia, Viçosa, v. 29, n. 4, p. 1091-1097, 2000.

BORGES, A. F.; OLIVEIRA, R. F. M.; DONZELE, J. L. Exigência de lisina para frangos de corte machos no período de 22 a 42 dias de idade, mantidos em ambiente quente $\left(26^{\circ} \mathrm{C}\right)$. Revista Brasileira de Zootecnia, Viçosa, v. 31, n. 5 , p. 1993-2001, 2002.

CELLA, P. S.; DONZELE, J. L.; OLIVEIRA, R. F. M. Planos de nutrição para frangos de corte no período de 1 a 49 dias de idade mantidos em condições de conforto térmico. Revista Brasileira de Zootecnia, Viçosa, v. 30, n. 2, p. 425432, 2001.

CONHALATO, G. S. Exigência de lisina digestível para frangos de corte machos. 1998. 79 f. Dissertação (Mestrado emZootecnia) - Universidade Federal de Viçosa, Viçosa, 1998.

COSTA, F. G. P. Níveis dietéticos de lisina e proteína bruta para frangos de corte. 2000. $156 \mathrm{f}$. Tese (Doutorado em Zootecnia) - Universidade Federal de Viçosa, Viçosa, 2000.

FARREL, C. L.; KOONG, K. J. Influence of plane nutrition on body composition, organ size and energy utilization of sprague-dawley rats. Journal of Nutrition, Philadelphia, n. 116, p. 2525-2535, 1986.

HAN, Y.; BAKER, D. H. Digestible lysine requirement of male and female broiler chicks during the period three to six weeks posthatching. Poultry Science, Champaign, v. 73, p. 1739-1745, 1994. 
LECLERCQ, B. Specifc effects of lysine on broiler production: comparison with threonine and valine. Poultry Science, Champaign, v. 77, p. 118-123, 1998.

NASCIMENTO, A. Lisina: principal aminoácido para deposição protéica. Revista Ave World, [S.l.], ano 1, n. 1, p. 56-61, 2003a.

NASCIMENTO, A. Lisina otimiza a conversão alimentar. Revista Ave World, [S.1.], ano 1, n. 5, p. 48-50, 2003 b.

NATIONAL RESEARCH COUNCIL. Nutrient requirements of poultry. 9 . ed. Washington, DC: National Academy, 1994. $155 \mathrm{p}$.

PACK, M. Proteína ideal para frangos de corte: conceitos e posição atual. In: CONFERÊNCIA APINCO DE CIÊNCIAS E TECNOLOGIAAVÍCOLAS, 1995, Curitiba. Anais... Curitiba: APINCO, 1995. p. 95-110.

REZENDE, J. A. A.; ROSTAGNO, H. S.; SILVA, M. A.; FONSECA, J. B.; COSTA, P. M. Níveis de proteína, aminoácidos sulfurosos e lisina em rações de frangos submetidos a regime de alta temperatura: fase final. Revista da Sociedade Brasileira de Zootecnia, Viçosa, v. 9, n. 1, p. 125-141, 1980.

ROSTAGNO, H. S.; DIONIZIO, M. A.; ALBINO, L. F. T. Perspectivas da nutrição de frangos de corte. In: REUNIÃO ANUAL DA SOCIEDADE BRASILEIRA DE ZOOTECNIA, 41., 2004, Campo Grande. Anais... Campo Grande: [s.n.], 2004. CD-ROM.
ROSTAGNO, H. S.; SILVA, D. J.; COSTA, P. M. A. Composição de alimentos e exigências nutricionais de aves e suínos: tabelas brasileiras. Viçosa: UFV, 2000. 141 p.

SCOTT, M. L.; NESHEIM, M. C.; YOUNG, R. J. Nutrition of the chicken. 3. ed. Ithaca: [s.n.], 1982. 562 p.

SKINNER, J. T.; IZALT, A. L.; WALDROUP, P. W. Effects of dietary amino acid levels on performance and carcass composition of broilers 42 to 49 days of age. Poultry Science, Champaign, v. 70, p. 1223-1230, 1992.

SAS INSTITUTE. SAS user guide: statistics. 12. ed. New York, 1996. $511 \mathrm{p}$.

SILVA, S. H. M.; ALBINO, L. F. T.; VARGAS JÚNIOR, J. G. Níveis de metionina + cistina para frangos de corte de 1 a 21 dias de idade. Revista Brasileira de Zootecnia, Viçosa, v. 28, n. 3, p. 519-525, 1999.

SUIDA, D. Formulação por proteína ideal e conseqüências técnicas, econômicas e ambientais. In: SIMPÓSIO INTERNACIONAL DE NUTRIÇÃOANIMAL: PROTEÍNA IDEAL, ENERGIA LÍQUIDA E MODELAGEM, 1., 2001. Santa Maria, RS. Anais... Santa Maria: [s.n.], 2001.

VALÉRIO, S. R.;OLIVEIRA, R. F. M.;DONZELE, J. L. Níveis de lisina digestível em rações, mantendo ou não a relação aminoacídica, para frangos de corte de 22 a 42 dias de idade, sob condições de estresse por calor. Revista Brasileira de Zootecnia, Viçosa, v. 32, n. 2, p. 372-382, 2003. 